\title{
Acquired somatic variants in inherited myeloid malignancies
}

\author{
Hannah Armes ${ }^{1,5}$, Ana Rio-Machin (DD ${ }^{1,5}{ }^{凶}$, Szilvia Krizsán (iD ${ }^{2}$, Csaba Bödör (D) ${ }^{2}$, Fadimana Kaya (D) ${ }^{1}$, Findlay Bewicke-Copley (D) ${ }^{1}$, \\ Jenna Alnajar D $^{3}$, Amanda Walne ${ }^{3}$, Borbála Péterffy ${ }^{2}$, Hemanth Tummala ${ }^{3}$, Kevin Rouault-Pierre (iD ${ }^{4}$, Inderjeet Dokal ${ }^{3}$, \\ Tom Vulliamy ${ }^{3}$ and Jude Fitzgibbon (iD ${ }^{1 凶}$ \\ (c) The Author(s) 2022
}

Leukemia (2022) 36:1377-1381; https://doi.org/10.1038/s41375-022-01515-2

\section{TO THE EDITOR:}

Inherited myeloid malignancies are considered a rare disease entity that exhibit significant heterogeneity in penetrance, age of onset and clinical presentation. When exploring disease evolution in this setting, determination of a causal germline variant represents one piece of the puzzle, yet the clinical heterogeneity we observe, even within families, suggests that germline carriers are also influenced by the acquisition of somatic mutations. Despite advances in the identification of germline variants, our understanding of the landscape of acquired mutations in familial myelodysplastic syndrome (MDS) and acute myeloid leukaemia (AML) remains incomplete.

We recently performed an analysis of the largest cohort of MDS/ AML families to date, utilising next-generation sequencing technologies to profile the coding genome and identify inherited lesions in families with a clinical history suggestive of germline involvement [1]. By looking at previously defined disease-causing loci, we identified putative pathogenic germline variants in $57 \%$ of families across 16 genes.

In the current study, we focused our attention on acquired mutations within a cohort of 33 well-characterised MDS/AML families where $\geq 2$ members were diagnosed with a haematological disorder, of which $\geq 1$ case was specified as MDS/AML. DNA samples corresponding to 51 individuals from these families were available for the study (Supplementary Fig. S1). Of these, 16 were diagnosed with AML, 22 MDS, 1 thrombocytopenia (TCP), 7 bone marrow failure (BMF), 1 lymphoedema, 1 had abnormal lymphocyte subsets and 3 were asymptomatic carriers (Supplementary Table S1, Supplementary Fig. S1). Each individual carried a germline variant in 1 of 13 discrete loci known to predispose to familial MDS/AML (Supplementary Fig. S2). The age of onset of MDS/AML was highly variable (median, 30 years; range, 1-76 years) and dependent on the nature of the germline mutations. RUNX1, CEBPA and GATA2 families exhibited early-onset MDS/AML, with a median age of 10, 18 and 21 years at presentation, respectively, compared with TERT/TERC, SRP72 and DDX41 families who had a longer latency, and a median age of 41.5, 51 and 56 years, respectively (Supplementary Fig. S3).

Acquired mutations were assessed using a commercial 54-gene TruSight Myeloid Sequencing Panel (Illumina; Supplementary Table S2), performed at a mean depth of 1,300 reads following the filtering criteria summarised in Supplementary Fig. S4 (data available in the European Nucleotide Archive; Accession Number: PRJEB49554). In total, 78 acquired mutations passed our filtering criteria, corresponding to 27 of the 54 genes analysed (Fig. 1; Supplementary Table S3), with 41 (53\%) of these catalogued as somatic mutations in COSMIC and 59 (76\%) absent in control populations. At least one acquired mutation was identified in 28/ 51 individuals including $10 \mathrm{AML}$ and $12 \mathrm{MDS}$ patients, and the median variant allele frequency (VAF) of the acquired mutations was $11.7 \%$ (range, $5.2-99.5 \%$ ).

To better define the spectrum of acquired mutations in inherited myeloid disorders, we performed an integrative analysis of the acquired mutational signature in our cohort in addition to published families harbouring germline variants in the same 13 predisposing loci. We have included $395 \mathrm{MDS} / \mathrm{AML}$ patients from across 64 publications (Supplementary Fig. S5; Supplementary Table S4), and even accounting for differences in patient inclusion, sequencing methodology and analysis we observed a high concordance between our results and previously published families.

The integrated results, summarised in Fig. S6, demonstrate the heterogeneity of acquired mutations that exists in familial myeloid malignancies. Overall, this analysis confirmed previous observations of the striking frequency of second-hit RUNX1, CEBPA and $D D X 41$ mutations in patients harbouring these germline variants, recurrence of monosomy 7 , trisomy 8 , and STAG2 and ASXL1 mutations in GATA2 families, and acquired GATA2 mutations secondary to germline CEBPA variants. We also observed a striking prevalence of acquired TP53 mutations in SBDS patients, with several individuals exhibiting multiple TP53 mutations, and a high frequency $(>80 \%)$ of DDX41-mutated cases presenting as cytogenetically normal (Supplementary Table S4). It is harder to apportion importance to many other acquired mutations as they arise infrequently and the size and number of patient cohorts remain low overall. That said, it is worth recognising the relevance of secondary variants in defining clinical manifestations in inherited malignancies, for instance, ETV6-mutated AML exhibits a distinct somatic profile to acute lymphoblastic leukaemia (ALL) even when arising from the same germline variant, suggesting that cooperating mutations can impact whether a neoplasm develops in the lymphoid or myeloid lineage [2].

Our study is the first to report the nature of acquired variants in SRP72 families (FML033: p.Thr355Lysfs*19; FML034: p.Arg207His). While both families exhibited similar clinical manifestations, presenting with BMF or TCP in childhood/early adulthood, with some individuals developing MDS in adulthood [3], we observed

\footnotetext{
${ }^{1}$ Centre for Genomics and Computational Biology, Barts Cancer Institute, Queen Mary University of London, London, UK. ${ }^{2}$ HCEMM-SE Lendulet Molecular Oncohematology Research Group, 1st Department of Pathology and Experimental Cancer Research, Semmelweis University, Budapest, Hungary. ${ }^{3}$ Centre for Genomics and Child Health, Blizard Institute, Queen Mary University of London, London, UK. ${ }^{4}$ Centre for Haemato-Oncology, Barts Cancer Institute, Queen Mary University of London, London, UK. ${ }^{5}$ These authors contributed equally: Hannah Armes, Ana Rio-Machin. ${ }^{凶}$ email: a.rio-machin@qmul.ac.uk; j.fitzgibbon@qmul.ac.uk
}

Received: 8 October 2021 Revised: 13 January 2022 Accepted: 26 January 2022

Published online: 9 February 2022 


\begin{tabular}{|c|c|c|c|c|c|c|c|c|c|c|c|c|c|c|c|c|c|c|c|c|c|c|c|c|c|c|c|c|}
\hline 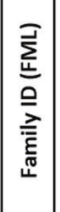 & 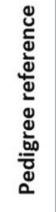 & 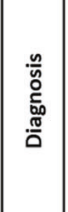 & 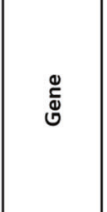 & 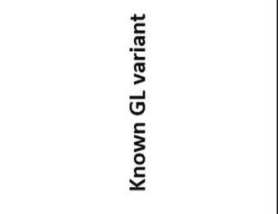 & 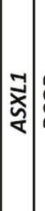 & & : & 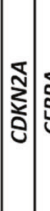 & 怘 & : & 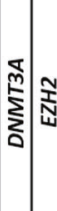 & 芯 & 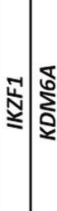 & 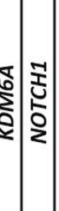 & 离 & $\frac{1}{\frac{1}{2}} \frac{7}{2}$ & ปิ & 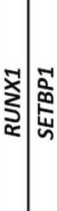 & 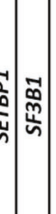 & ڤ్రి & 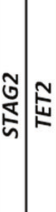 & $\underline{\underline{y}}$ & గ్ & 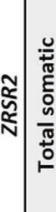 & 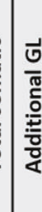 & 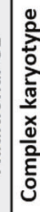 & 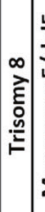 & 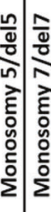 \\
\hline 001 & II.1 & AML & \multirow{7}{*}{$\begin{array}{l}\text { ¿ } \\
\text { 岂 }\end{array}$} & p.Glu50Alafs*104 & & & & & & & & & & & & & & & & & & & & 0 & 1 & & & \\
\hline 001 & 11.2 & AML & & p.Glu50Alafs*104 & & & & & & & & & & & & & & & & & & & & 1 & 1 & & & \\
\hline 002 & 11.2 & AML & & p.His24Alafs*84 & & & & & & & & & & & & & & & & & & & & 1 & 0 & & & \\
\hline \begin{tabular}{|l|}
002 \\
\end{tabular} & 1.1 & AML & & p.His24Alafs $* 84$ & & & & & & & & & & & & & & & & & & & & 1 & 0 & & & \\
\hline 002 & 1.1 & AML & & p.His24Alafs*84 & & & & & & & & & & & & & & & & & & & & 4 & 0 & & & \\
\hline 004 & II.1 & AML & & p.Pro23Argfs*137 & & & & & & & & & & & & & & & & & & & & 1 & 0 & & & \\
\hline 004 & 11.5 & AML & & p.Pro23Argfs*137 & & & & & & & & 2 & & & & & & & & & & & & 2 & 0 & & & \\
\hline 013 & 11.2 & MDS & DDX41 & p.Arg124* & & & & & & & & & & & & & & & & & 2 & 2 & & 3 & 0 & & & \\
\hline 042 & III.1 & BMF & ERCC6L2 & p.Glu923Argfs & & & & & & & & & & & & & & & & & & & & 1 & 0 & & & \\
\hline 014 & IV.2 & AML & ETV6 & p.Leu117Phe & & & & & & & & & & & & & & & & & & & & 2 & 0 & & & \\
\hline 044 & 11.2 & $\mathrm{AA}$ & FANCA & c. $2505-1 G>T / c .3626+5 G>C$ & & & & & & & & & & & & & & & & & & & & 1 & 0 & & & \\
\hline 016 & III.3 & MDS & \multirow{5}{*}{ 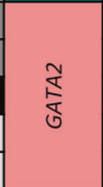 } & p.Gly28fs*/p.His26Pro & & & & & & & & & & & & & & & & & & & & 1 & 0 & & & \\
\hline 019 & 11.1 & MDS & & p.Arg362* & & & & & & & & & & & & & & & & & & & & 2 & 0 & & & \\
\hline 021 & II.1 & AML & & p.Leu105Profs*15 & & & & & & & & & & & & & & & & & & & & 4 & 0 & & & \\
\hline 021 & 1.2 & $A b . L$ & & p.Leu105Profs*15 & & & & & & & & & & & & & & & & & & & & 1 & 0 & & & \\
\hline 021 & 1.3 & $L$ & & p.Leu105Profs*15 & & & & & & & & & & & & & & & & & & & & 2 & 0 & & & \\
\hline 045 & 11.2 & MDS & MECOM & p.Arg815Trp & & & & & & & & & & & & & & & & & 2 & 2 & & 4 & 0 & 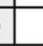 & & \\
\hline 027 & 11.2 & AML & \multirow{3}{*}{$\underset{\gtrless}{\mathbb{x}}$} & p.Asp123His & & & & & & & & & & & & & & & & & & & & 2 & 0 & & & \\
\hline 029 & III.4 & TCP & & Del & & & & & & & & & & & & & & & & & & & & 1 & 0 & & & \\
\hline 088 & NA & MDS & & Del & & & & & & & & & & & & & & & & & & & & 1 & 0 & & & \\
\hline 047 & 11.2 & MDS & SBDS & p. Lys $62 * / c .258+2 T>C$ & & & & & & & & & & & & & & & & & & 2 & & 3 & 0 & 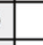 & & \\
\hline 033 & II.2 & BMF & \multirow{4}{*}{ 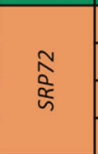 } & p.Thr355Lysfs*19 & & & & & & & & & & & & & & & & & & & & 0 & 1 & 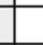 & & \\
\hline 033 & 11.3 & BMF & & p.Thr355Lysfs*19 & & & & & & & & & & & & & & & & & & & & 0 & 1 & . & & \\
\hline 034 & 11.2 & MDS & & p.Arg207His & & & & & & & & & & & & & & & & & & & & 1 & 0 & & & \\
\hline 034 & 1.1 & MDS & & p.Arg207His & & & & & & & & & & & & & 2 & & & & & & & 6 & 0 & 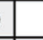 & & \\
\hline 035 & 11.2 & MDS & \multirow{2}{*}{ TERC } & $r .212 C>G$ & & & & & & & & & & & & & & & & & & & & 5 & 0 & & & \\
\hline 036 & 111.3 & MDS & & $r .309 G>T$ & & 2 & & & & & & & & & & & & & & & & & & 3 & 0 & & & \\
\hline 039 & III.1 & $A A$ & \multirow{6}{*}{ 虑 } & p.Arg631Gln & & 2 & 2 & & & & & & 2 & 2 & & & & & & & & & & 13 & $\begin{array}{ll}3 & 0 \\
\end{array}$ & 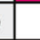 & & \\
\hline 040 & $\mathrm{I} .1$ & $A C$ & & p.Pro785Leu & & & & & & & & & & & & & & & & & & & & 0 & 1 & 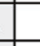 & & \\
\hline 040 & 11.1 & $\mathrm{AA}$ & & p.Pro785Leu & & & & & & & & & & & & & & & & & & & & 0 & 2 & ? & & \\
\hline 040 & II.2 & $A C$ & & p.Pro785Leu & & & & & & & & & & & & & & & & & & & & 0 & 1 & & & \\
\hline 040 & 11.4 & AML & & p.Pro785Leu & & & & & & & & & & 3 & & & & & & & & 3 & & 10 & $\begin{array}{ll}0 & 1 \\
\end{array}$ & & & \\
\hline 041 & III. 4 & MDS & & p.His482Profs*27 & & & & & & & & & & & & & & & & & & & & 1 & 0 & 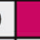 & & \\
\hline 030 & III.1 & MDS & TPP1 & p.Lys170del & & & & & & & & & & & & & & & & & & & & 1 & 0 & & & \\
\hline
\end{tabular}

Key

Acquired variant, VAF $>10 \%$

Acquired variant, VAF $<10 \%$

Germline variant

Karyotypic abnormality

Fig. 1 Aggregation of the acquired mutations identified in our series of patients. Only patients with one or more acquired or additional germline variant in our series are included. Purple represents mutations with a VAF $>10 \%$; turquoise, VAF $<10 \%$; black, additional germline variants and pink represents karyotypic abnormalities. Abbreviations: AA aplastic anaemia, Ab. L abnormal lymphocyte subsets, AC asymptomatic carrier, AML acute myeloid leukaemia, BMF bone marrow failure, GL germline, L lymphoedema, MDS myelodysplastic syndrome. For further details of somatic mutations in our series refer to Supplementary Table S3.

inter- and intra-familial heterogeneity in their mutational profiling. Two siblings from FML033 (II.2, II.3) with congenital nerve deafness and presenting with BMF at 12 and 11 years, respectively, harboured an EZH2 germline variant (p.Met191Val, uncertain significance, gnomAD frequency: $8.8 \times 10^{-6}$ ) but no cooperating acquired mutations. In FML034, the index case (II.2) was diagnosed with refractory anaemia with excess blasts (MDS-RAEB) at 51 years and harboured a single KDM6A variant (p.Ser1192Pro, absent in gnomAD; $V A F=50 \%$, predicted pathogenic), while her mother
(I.1), who also developed MDS in her 70's, harboured six low-VAF secondary variants (ASXL1, CSF3R, GATA2, IKZF1 and two variants in $R A D 21$; all with $\mathrm{VAF}<10 \%)$ (Supplementary Table S3).

Importantly, our study also identifies a novel mutational signature in TERT families for the first time. Four families with germline TERT variants were included in our series (FML038, FML039, FML040 and FML041), corresponding to six patients with aplastic anaemia (AA) $(N=2), \operatorname{MDS}(N=3)$ and $\operatorname{AML}(N=1)$, and three asymptomatic carriers. Fig. $2 A$ shows a schematic of the 
A

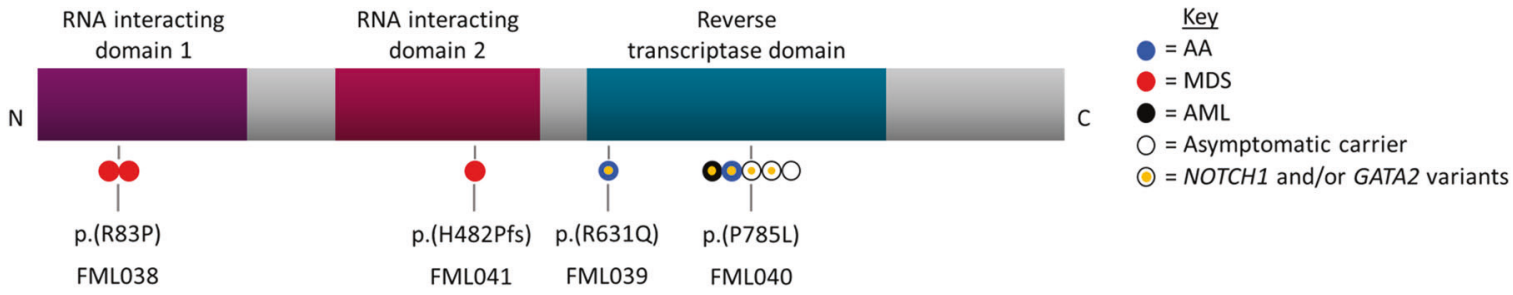

B

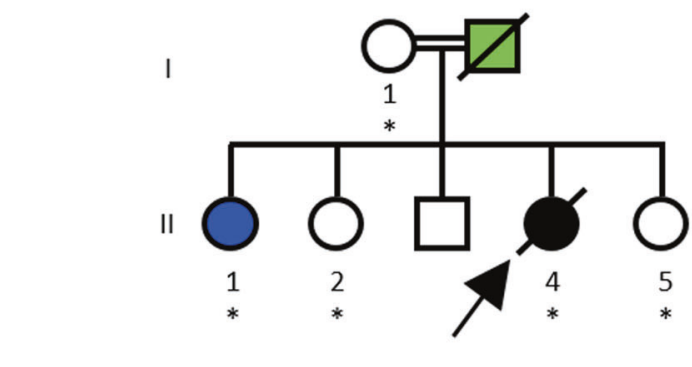

C

FML039

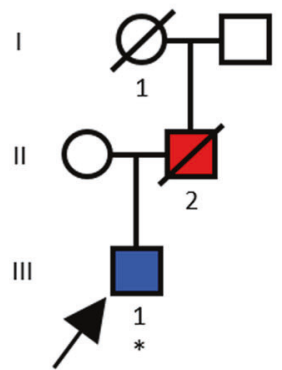

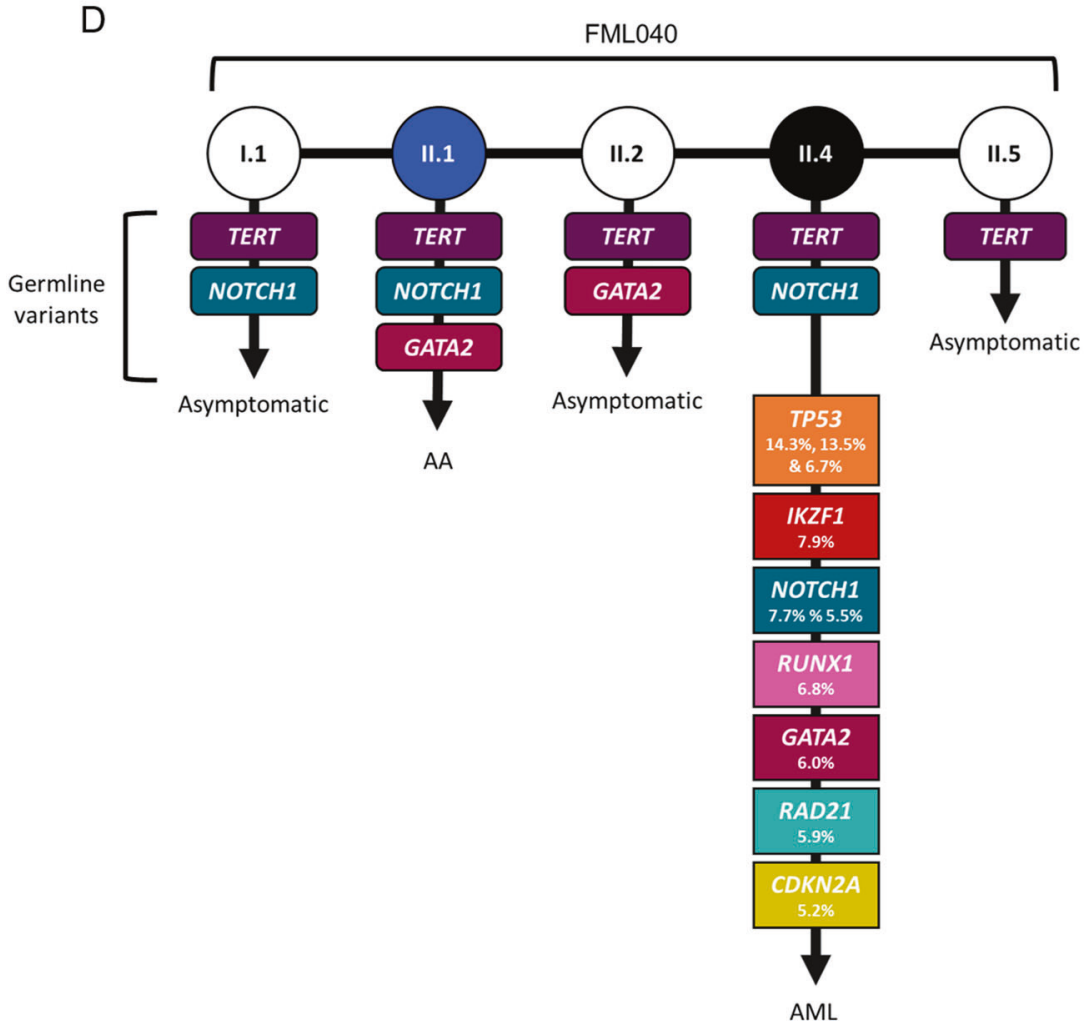

FML039

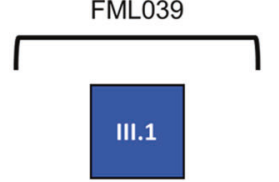

TERT
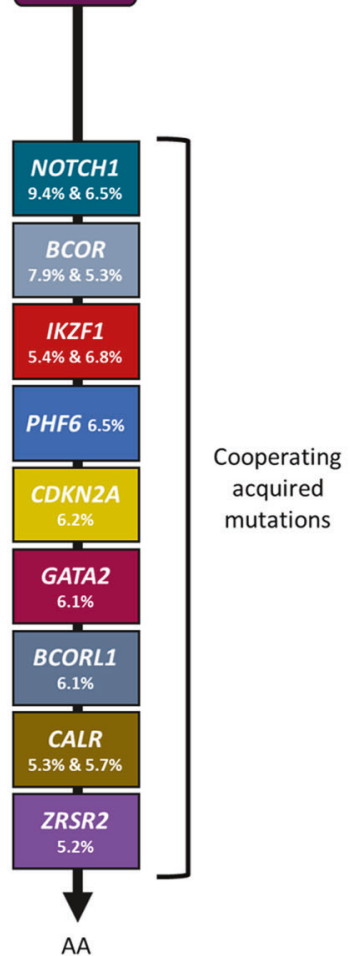

Fig. 2 Analysis of TERT families. A Schematic of the TERT protein. Location of germline variants of 9 individuals in our series are depicted in relation to the RNA-interacting and catalytic domain(s). Circles represent individual cases and are colour coded by disease phenotype. B, C Schematic representation of TERT families, FML040 and FML039. Black represents AML; red, MDS; blue, bone marrow failure and green, other cancer. Samples included for targeted sequencing are indicated with an asterisk. In FML040, individuals I.1, II.2 and II.5 are asymptomatic carriers of the TERT germline variant. In FML039, individual I.1 is also an asymptomatic carrier. D Variants identified in TERT families, FMLO40 and FML039. Germline variant pattern (TERT, GATA2, NOTCH1), cooperating acquired mutations with corresponding VAF and patient phenotype (AML, AA or asymptomatic) are indicated. 
location of the four TERT germline variants: FML038 (p.Arg83Pro) and FML041 (p.His482Profs*27) harbour heterozygous missense and truncating variants, respectively, within the N-terminal RNAinteracting domains, while families FML039 (p.Arg631Gln) and FML040 (p.Pro785Leu) (Fig. 2B, C) retained heterozygous missense TERT variants located within the C-terminal catalytic reverse transcriptase domain (RTD) responsible for maintaining telomere ends. Carriers of the RTD TERT variants in FML039 and FML040 were previously found to exhibit short telomeres and abolished/ reduced telomerase activity [4].

We observed a complex pattern of inheritance in FML040 suggesting that the clinical presentation in this family may arise from germline variants at three discrete loci, including TERT, NOTCH1 and GATA2 (Fig. 2D; Supplementary Fig. S7). TERT and NOTCH1 (p.Pro2128Leu, uncertain significance, gnomAD frequency: $7 \times 10^{-5}$ ) variants were inherited from the asymptomatic mother in two offspring, including the index case who developed secondary AML (II.4), and her sister with a diagnosis of AA (II.1). Sibling II.1 also inherited a germline GATA2 variant (p.Pro14Ser; predicted damaging in SIFT and MutationTaster, gnomAD frequency: $4.8 \times 10^{-5}$ ) that was also present in her asymptomatic sister (II.2) which we presume was inherited from her father (I.II). The genetic pattern is further complicated by the existence of acquired mutations in NOTCH1 (VAF $=5.5 \%$ p.Glu1636Lys, gno$\mathrm{mAD}$ frequency: $1.29 \times 10^{-5}, \operatorname{COSM} 308616$; and $\mathrm{VAF}=7.7 \% \mathrm{p}$. Ala1634Asp, absent in gnomAD, COSM308589) and GATA2 (VAF = $6.0 \%$ p.Phe400Leu, absent in gnomAD and COSMIC) at low VAF in II.4. Therefore, in our current model we propose that the onset of overt symptoms may be conditional on the presence of both NOTCH1 and GATA2 variants, since the mother (I.1) and sibling (II.2) remain symptom-free and harbour a single NOTCH1 or GATA2 variant, respectively.

In support of this model, we found that the index case in family FML039 (III.1), with AA and harbouring a germline TERT variant in the RTD, also retained two acquired NOTCH1 mutations (VAF = $6.5 \%$ p.Pro $2551 \mathrm{fs}$, absent in gnomAD, COSM6918461; and $\mathrm{VAF}=$ 9.4\% p.Ser2499delinsSerPro, absent in gnomAD and COSMIC) and a GATA2 mutation (VAF $=6.1 \%$ p.Glu398Lys, absent in gnomAD, COSM7119678). It is plausible that these low VAF mutations represent a subclonal population of cells that may serve as a reservoir population from which transformed cells later emerge. The combination of NOTCH1 and GATA2 together may act as a risk factor for development of myeloid disease when in conjunction with the germline TERT variant. Though the precise molecular mechanism for the functional interaction between TERT, NOTCH1 and GATA2 is open to speculation, telomerase has previously been shown to impact NOTCH1 signalling [5] while NOTCH1 directly regulates the expression of GATA2 during endothelial-tohaematopoietic transition during development [6]. We also noted co-occurrence of somatic IKZF1 and CDKN2A mutations with NOTCH1 in the two index cases of the TERT families (FML039, FML040). This may not be surprising, since IKZF1 appears to cooperate with NOTCH1 pathway activation to maintain homoeostasis of monocytic/dendritic progenitors [7] and T-ALLactivating Notch 1 mutations in mice frequently coincide with loss-of-function mutations in Ikzf1 [8-11]. This suggests there is value in reassessing other TERT families, particularly those with a germline RTD variant, to determine whether additional NOTCH1 and GATA2 mutations are a wider feature of these presentations or indeed, if other related mutations can also be detected in these families.

Altogether, we provide a comprehensive analysis of the somatic mutational landscape across a range of BMF and MDS/AML families, integrating the somatic profiling of 446 individuals from this study and previously published families with inherited variants in 13 gene loci. Our analysis demonstrates the importance of consortium efforts, championed by the RUNX1 foundation and Brown and colleagues [12], which enable trends of mutational acquisition to be identified in large cohorts of familial patients. Our dataset has provided insights into the complex interplay between genetic lesions that may contribute to disease development, particularly in the case of TERT-mutated families, suggesting that disease progression may be shaped by a particular combination of germline and somatic co-occurring variants. Above all, there is an unmet need to include familial loci for analysis in routine diagnostic panels, not only to document instances of inherited disease, but also to provide a resource to investigate acquired mutations and gain a greater understanding of the pathogenesis of both familial and sporadic disease.

\section{REFERENCES}

1. Rio-Machin A, Vulliamy T, Hug N, Walne A, Tawana K, Cardoso S, et al. The complex genetic landscape of familial MDS and AML reveals pathogenic germline variants. Nat Commun. 2020;11:1044.

2. Nishii R, Baskin-Doerfler R, Yang W, Oak N, Zhao X, Yang W, et al. Molecular basis of ETV6-mediated predisposition to childhood acute lymphoblastic leukemia. Blood. 2021;137:364-73.

3. Kirwan M, Walne AJ, Plagnol V, Velangi M, Ho A, Hossain U, et al. Exome sequencing identifies autosomal-dominant SRP72 mutations associated with familial aplasia and myelodysplasia. Am J Hum Genet. 2012;90:888-92.

4. Kirwan M, Vulliamy T, Marrone A, Walne AJ, Beswick R, Hillmen P, et al. Defining the pathogenic role of telomerase mutations in myelodysplastic syndrome and acute myeloid leukemia. Hum Mutat. 2009;30:1567-73.

5. Theodoris CV, Mourkioti F, Huang Y, Ranade SS, Liu L, Blau HM, et al. Long telomeres protect against age-dependent cardiac disease caused by NOTCH1 haploinsufficiency. J Clin Investig. 2017;127:1683-8.

6. Lomelí $\mathrm{H}$, Castillo-Castellanos F. Notch signaling and the emergence of hematopoietic stem cells. Dev Dyn. 2020;249:1302-17.

7. Mastio J, Simand C, Cova G, Kastner P, Chan S, Kirstetter P. Ikaros cooperates with Notch activation and antagonizes TGF $\beta$ signaling to promote pDC development. PLoS Genet. 2018;14:e1007485.

8. Witkowski MT, Cimmino L, Hu Y, Trimarchi T, Tagoh H, McKenzie MD, et al. Activated Notch counteracts Ikaros tumor suppression in mouse and human T-cell acute lymphoblastic leukemia. Leukemia. 2015;29:1301-11.

9. Beverly LJ, Capobianco AJ. Perturbation of Ikaros isoform selection by MLV integration is a cooperative event in Notch(IC)-induced T cell leukemogenesis. Cancer Cell. 2003;3:551-64.

10. López-Nieva P, Santos J, Fernández-Piqueras J. Defective expression of Notch1 and Notch2 in connection to alterations of c-Myc and lkaros in gamma-radiationinduced mouse thymic lymphomas. Carcinogenesis. 2004;25:1299-304.

11. Uren AG, Kool J, Matentzoglu K, de Ridder J, Mattison J, van Uitert M, et al. Largescale mutagenesis in p19(ARF)- and p53-deficient mice identifies cancer genes and their collaborative networks. Cell. 2008;133:727-41.

12. Brown AL, Arts $P$, Carmichael $C L$, Babic M, Dobbins J, Chong CE, et al. RUNX1mutated families show phenotype heterogeneity and a somatic mutation profile unique to germline predisposed AML. Blood Adv. 2020;4:1131-44.

\section{ACKNOWLEDGEMENTS}

The authors are grateful to the patients and their families for donating specimens for research in this study. This study was funded by Blood Cancer UK (14032), Cancer Research UK (C15966/A24375) and the Medical Research Council (MR/P018440/1). CB, SK and BP were supported by the Hungarian National Research, Development and Innovation Office (ED ED18-1-2019-0019, TKP2021-EGA-24 and FK20-134253) and the EU's Horizon 2020 Research and Innovation Program (739593). FK was supported by Republic of Turkey Ministry of National Education, General Directorate of Higher and Foreign Education, Turkish Government Study Abroad Program.

\section{AUTHOR CONTRIBUTIONS}

$J F, H A, A R-M, I D$ and TV devised the project, designed the experiments and wrote the manuscript. HA, AR-M, F.K and HT performed research and collected data. JA, AW and KR-P contributed to data collection. SK, CB and BP performed targeted sequencing. AR-M, TV, HA and FB-C performed data analysis.

\section{COMPETING INTERESTS}

The authors declare no competing interests. 


\section{ADDITIONAL INFORMATION}

Supplementary information The online version contains supplementary material available at https://doi.org/10.1038/s41375-022-01515-2.

Correspondence and requests for materials should be addressed to Ana Rio-Machin or Jude Fitzgibbon.

Reprints and permission information is available at http://www.nature.com/ reprints

Publisher's note Springer Nature remains neutral with regard to jurisdictional claims in published maps and institutional affiliations.
Open Access This article is licensed under a Creative Commons Attribution 4.0 International License, which permits use, sharing, adaptation, distribution and reproduction in any medium or format, as long as you give appropriate credit to the original author(s) and the source, provide a link to the Creative Commons license, and indicate if changes were made. The images or other third party material in this article are included in the article's Creative Commons license, unless indicated otherwise in a credit line to the material. If material is not included in the article's Creative Commons license and your intended use is not permitted by statutory regulation or exceeds the permitted use, you will need to obtain permission directly from the copyright holder. To view a copy of this license, visit http://creativecommons. org/licenses/by/4.0/.

(c) The Author(s) 2022 